\title{
Postoperative pulmonary edema in a patient with POEMS syndrome
}

\author{
Yoonji Jo ${ }^{1}$, Jee-Eun Chang ${ }^{2}$, Seokha Yoo ${ }^{1}$, and Jin Huh $^{2}$ \\ Department of Anesthesiology and Pain Medicine, ${ }^{1}$ Seoul National University Hospital, ${ }^{2}$ SMG-SNU Boramae Medical Center, Seoul, Korea
}

POEMS syndrome is a rare multisystemic disorder. It is characterized by polyneuropathy, organomegaly, endocrinopathy, monoclonal gammopathy, and skin changes [1]. Because it is also associated with pulmonary dysfunction, a patient with POEMS syndrome may have postoperative respiratory difficulty. In this report, we describe a patient with this syndrome who underwent surgery under general anesthesia and failed ventilator weaning due to pulmonary edema.

A 58-year-old male (height $163 \mathrm{~cm}$; weight $48.5 \mathrm{~kg}$ ) with POEMS syndrome was diagnosed with rectal cancer and planned to undergo low anterior resection. He displayed weakness and paresthesia of the right arm and both legs, skin lesions such as hypertrichosis, scleroderma, and thickened extremities, and orthopnea, which had developed 2 weeks previously. Chest radiography showed bilateral mild pleural effusion. A pulmonary function test $(\mathrm{PFT})$ revealed a mild restrictive pattern. Arterial blood gas analysis (ABGA) in room air was $\mathrm{pH} 7.398$, partial carbon dioxide pressure $\left(\mathrm{PaCO}_{2}\right)$ was $27.8 \mathrm{mmHg}$, partial oxygen pressure $\left(\mathrm{PaO}_{2}\right)$ was $91.4 \mathrm{mmHg}$, and bicarbonate $\left(\mathrm{HCO}^{3-}\right)$ concentration was $16.8 \mathrm{mEq} / \mathrm{L}$. On echocardiogram, the estimated pulmonary artery systolic pressure (PASP) was $40 \mathrm{mmHg}$, and the other values were normal. Other laboratory data and vital signs were within normal limits.

Anesthesia was induced with propofol $120 \mathrm{mg}$, fentanyl 100 $\mu \mathrm{g}$, and rocuronium $50 \mathrm{mg}$. A size 7.5, cuffed tube was used for intubation. A radial arterial line and a central venous catheter were placed in the right internal jugular vein. Anesthesia was maintained with sevoflurane in air $/ \mathrm{O}_{2}$. Thirty minutes into the surgery, systolic blood pressure (BP) decreased to 70-80 $\mathrm{mmHg}$.
Continuous infusion of dopamine and norepinephrine was initiated. An additional $5 \mathrm{mg}$ of rocuronium were injected four times. The last ABGA intraoperatively was $\mathrm{pH} 7.263, \mathrm{PaCO}_{2}$ $40.3 \mathrm{mmHg}, \mathrm{PaO}_{2} 96.0 \mathrm{mmHg}, \mathrm{HCO}^{3-} 17.8 \mathrm{mEq} / \mathrm{L}$ under $\mathrm{FiO}_{2}$ 0.5 . The surgery duration was $190 \mathrm{~min}$ and the anesthesia time was $270 \mathrm{~min}$. The total amount of administered fluid was 1500 $\mathrm{ml}$ of crystalloid and $300 \mathrm{ml}$ of colloid solution. One and a half units of packed RBCs were transfused. Estimated blood loss was $400 \mathrm{ml}$, and urine output was $150 \mathrm{ml}$. Postoperatively, the patient was transferred to the intensive care unit (ICU).

On arrival at the ICU, his vital signs were $\mathrm{BP}, 132 / 58 \mathrm{mmHg}$; heart rate, 70 beats/min; and oxygen saturation, $100 \%$. Continuous infusion of dopamine $5 \mu \mathrm{g} / \mathrm{kg} / \mathrm{min}$ and norepinephrine $0.01 \mu \mathrm{g} / \mathrm{kg} / \mathrm{min}$ was maintained. No additional neuromuscular blocking agents were injected. On the second postoperative day (POD), desaturation was not detected after self-respiration with $\mathrm{O}_{2} 6 \mathrm{~L}$ for $3 \mathrm{~h}$, and the patient was extubated. Six hours after extubation, oxygen saturation decreased and the dyspnea appeared. The patient was reintubated and mechanical ventilation was initiated. Thoracic radiography revealed diffuse haziness and parahilar consolidation in both lung fields (Fig. 1). On the seventh POD, the patient was extubated again but was reintubated due to desaturation and dyspnea. On the $10^{\text {th }} \mathrm{POD}$, the haziness and consolidation on simple X-ray decreased, but the third extubation attempt on the $19^{\text {th }}$ POD also failed. Finally on the $27^{\text {th }}$ POD, he was weaned from the ventilator successfully. Four days after extubation, he was transferred to the ward.

Various respiratory manifestations are seen in patients with POEMS syndrome. Relatively common manifestations are pleu-

Corresponding author: Jin Huh, M.D., Department of Anesthesiology and Pain Medicine, SMG-SNU Boramae Medical Center, 20, Boramae-ro 5-gil, Dongjak-gu, Seoul 156-707, Korea. Tel: 82-2-870-2514, Fax: 82-2-846-2985, E-mail: huhjin419@gmail.com

(c) This is an open-access article distributed under the terms of the Creative Commons Attribution Non-Commercial License (http:// creativecommons.org/licenses/by-nc/3.0/), which permits unrestricted non-commercial use, distribution, and reproduction in any medium, provided the original work is properly cited. 


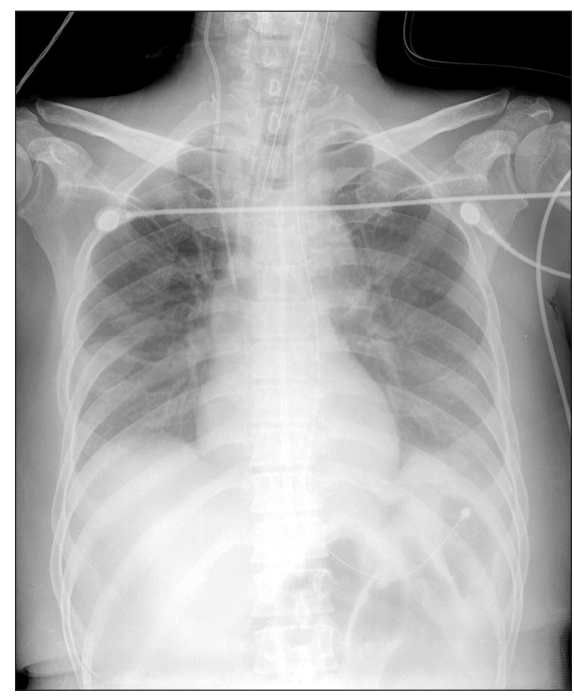

Fig. 1. Chest radiography on the second postoperative day.

ral effusion, pulmonary hypertension, restrictive lung disease, and a reduced diffusion capacity of carbon dioxide. Pulmonary tumors and phrenic neuropathy are less common [2,3]. Weakness of respiratory muscles also contributes to the pulmonary symptoms.

The patient in this report had several pulmonary manifestations-muscle weakness, pleural effusion, marginally high PASP, and restrictive pattern on PFT. Hence, in case he required postoperative respiratory support, we prepared the ICU before starting the surgery.

Ifuku et al. [4] reported that POEMS syndrome patients had a higher sensitivity to vecuronium and that the effects of vecuronium persisted longer. Therefore, during the anesthesia of POEMS syndrome patients, neuromuscular blocking agents should be carefully used with neuromuscular monitoring, or should be avoided when possible.

In this case, we used rocuronium, $50 \mathrm{mg}$ during induction and four doses of $5 \mathrm{mg}$ each during surgery. We attempted to use the lowest possible dose of muscle relaxants, but because self-respiration was detected and the surgeon reported abdomi- nal muscle tension, we used a small amount of additional rocuronium.

During induction of anesthesia, we checked the train of four (TOF) using nerve stimulators (Ministim ${ }^{\circledR}$ MS-IV, Life-Tech, Williston, Vermont, USA and E-NMT-OO, GE Healthcare Finland Oy, Helsinki, Finland) but the twitching was not detected. It is thought that severe subcutaneous edema and skin thickening blocked transmission of electric stimulation, which prevented observation of proper muscle contraction. Tschida et al. [5] reported that in patients with edematous and dry skin, TOF responses were inappropriate and did not correlate with the clinical assessment.

There were no significant findings on preoperative cardiac evaluations. However, after the surgery began, persistent hypotension was observed and inotropics were infused continuously. We infused $1500 \mathrm{ml}$ of crystalloid and $300 \mathrm{ml}$ of colloid to maintain blood pressure, and large amounts of fluid and blood were infused in the ICU. Such fluid infusion could aggravate pulmonary edema.

After the second failed attempt at extubation, pulmonology and neurology specialists were contacted to determine the cause of failure. Nerve conduction studies and an electromyogram could not be performed due to severe pitting edema, but the neurologist suggested that the dyspnea was not due to a neurological problem since no focal neurologic deficits were observed. In addition, the $6 \mathrm{~kg}$ weight gain after ICU admission, severe pitting edema, and chest radiography suggested that pleural effusion and pulmonary edema were the main causes of the failure.

In the ICU, diuretics were used to manage the pulmonary edema, but it was difficult to balance input and output because the blood pressure dropped easily with a small volume deficit. Ventilator weaning was finally accomplished on the $27^{\text {th }}$ POD.

In summary, because the POEMS syndrome may be accompanied by pulmonary dysfunction leading to postoperative respiratory distress, fluids should be managed judiciously and neuromuscular blocking agents should be used cautiously in anesthetic management.

\section{References}

1. Dispenzieri A. POEMS syndrome: update on diagnosis, risk-stratification, and management. Am J Hematol 2012; 87: 804-14.

2. Allam JS, Kennedy CC, Aksamit TR, Dispenzieri A. Pulmonary manifestations in patients with POEMS syndrome: a retrospective review of 137 patients. Chest 2008; 133: 969-74.

3. Mokhlesi B, Jain M. Pulmonary manifestations of POEMS syndrome: case report and literature review. Chest 1999; 115: 1740-2.

4. Ifuku Y, Minami K, Sata T, Inoue Y, Fujii K, Shigematsu A. Prolonged effects of vecuronium in a patient with Crow-Fukase syndrome. Masui 1999; 48: 424-6.

5. Tschida SJ, Hoey LL, Vance-Bryan K. Inconsistency with train-of-four monitoring in a critically ill paralyzed patient. Pharmacotherapy 1995; 15: 540-5. 